\title{
ONE PARAMETRIC MOTION IN KLEIN SPACES
}

\author{
MINORU KURITA
}

On the euclidean plane one-parametric motion is in general a roulett motion, exceptions being a translation at each instant and a rotation with a fixed center; here we mean by a roulett motion a motion in which a certain curve rolls on another fixed curve without slipping. In this paper we extend this fact to the case of Klein spaces and investigate in detail especially the cases of the euclidean space and the projective space. We repeatedly refer to

1. E. Cartan: La théorie des groupes finis et continus et la géométrie différentielle.

1. Let the fundamental Lie-group and its element be respectively $G$ and $S_{a}$, and let the fundamental frame be $R$. Take the frames $S_{a} T R$ and $S_{b} T R$, which are relatively fixed to $S_{a} R$ and $S_{b} R$ respectively. Then the transformation which shifts $S_{a} T R$ to $S_{b} T R$ is $\left(S_{a} T\right)^{-1} S_{b} T=T^{-1} S_{a}{ }^{-1} S_{b} T$. If $T^{-1} S_{a}^{-1} S_{b} T$ is an element of a certain subgroup $H$ of $G$, the set of frames $H R$ is invariant by the motion $T^{-1} S_{a}{ }^{-1} S_{b} T$. This $H R$ can be considered as an element of the homogeneous space that is generated by the cosets of $H$. This process is a generalization of the fact that the motion on the euclidean plane is either a translation or a rotation.

Now we consider a one-parametric motion $S_{a}$ in a Klein space, which we assume to be differentiable to the order we need. Put $\delta S=S_{a^{-1}} S_{a+d a}$ and let $T_{a}$ be a transformation such that $T_{a^{-1}} \delta S T_{a}$ belongs to a fixed subgroup $H$ of $G$, which is independent of $a$. We call $S_{a} T_{a} R$ the instantaneous center of this motion at an instant $a$. Then for the relative displacement $\delta(S T)$ of the instantaneous center we have

$$
\delta(S T)=\left(S_{a} T_{a}\right)^{-1}\left(S_{a+d a} T_{a+d a}\right)=T_{a}^{-1} \cdot \delta S \cdot T_{a} \cdot \delta T,
$$

where we put $\delta T=T_{a}{ }^{-1} T_{a+d a} . T_{a} R$ is the position of the instantaneous center relative to the moving frame $S_{a} R$, and $\delta T$ is the relative displacement of the instantaneous center on the moving space. Then we consider the relative components $\omega_{i}(a, d a)$ of $\delta S=S_{a^{-1}} S_{a+d a}$. As the relative components of the product of two infinitesimal motions are the sum of those of each motion, we get from (1) the result that the relative components of $\delta(S T)$ are equal to the sum of those of $T_{a}^{-1} \cdot \delta S \cdot T_{a}$ and $\delta T$. If we take the homogeneous space with cosets of

Received December 11, 1949. 
$H$ as its elements and consider the principal relative components of this homogeneous space, then the principal relative components of $\delta(S T)$ are equal to those of $\delta T . \quad S_{a} T_{a} R$ generate in the fixed space a one-parametric figure $C_{1}$ (basic figure) with cosets of $H$ as its elements, and similarly $T_{a} R$ generate a one-parametric figure $C_{0}$ (rolling figure) in the moving space. From the above consideration we get:

Theorem. Let $S_{a}$ be a one-parametric motion, for which there exists such $T_{a}$ that $T_{a}^{-1} \cdot \delta S \cdot T_{a}$ belongs to a fixed subgroup $H$ of the fundamental group $G$. Then the motion $S_{a}$ can be interpreted as the motion which is realized by the rolling of a certain figure $C_{0}$ along another figure $C_{1}$ without slipping. Here $C_{0}$ and $C_{1}$ are figures generated by the one-parametric set of cosets of $H$, and the rolling without slipping means that the principal components of $C_{0}$ and $C_{1}$ coinside at every instant with certain choice of frames attached to each elements of $C_{0}$ and $C_{1}$.

\section{Spherical Motion}

Let the orthogonal axes be $I_{1}, I_{2}, \ldots, I_{n}$, and their relative displacement be $d I_{i}=\sum_{j} \omega_{i j} I_{j}\left(\omega_{i j}+\omega_{j i}=0\right)$ which we write $d I=\Omega I$. By the coordinate transformation $\bar{I}=P I$ ( $P$, orthogonal), $\Omega$ is transformed to $\bar{\Omega}=P \Omega P^{\prime}$. If we take $P$ suitably, we have

$$
\bar{\Omega}=0+0+\ldots+0+\left(\begin{array}{lr}
0 & \lambda_{1} \\
-\lambda_{1} & 0
\end{array}\right)+\ldots+\left(\begin{array}{lr}
0 & \lambda_{k} \\
-\lambda_{k} & 0
\end{array}\right) .
$$

So we may take as the subgroup $H$ the one which consists of the transformations

$$
1+1+\ldots+1+\left(\begin{array}{ll}
\cos \theta_{1} & \sin \theta_{1} \\
-\sin \theta_{1} & \cos \theta_{1}
\end{array}\right)+\ldots+\left(\begin{array}{ll}
\cos \theta_{k} & \sin \theta_{k} \\
-\sin \theta_{k} & \cos \theta_{k}
\end{array}\right),
$$

and the instantaneous center is the set of points

$$
(1,0, \ldots, 0),(0,1,0, \ldots, 0), \ldots,(0,0, \ldots, 1,0, \ldots, 0),
$$

and several two dimensional planes. The one-parametric spherical motion is the one by which a one-parametric figure consisting of the above elements rolls on another figure of the same kind.

As an example we take the case $n=4$. Then an instantaneous center is a two-dimensional Grassmann manifold and if we take along the one-parametric set of these elements the frame $I_{1}, I_{2}, I_{3}, I_{4}$ suitably and put $d I_{i}=\sum_{j} \alpha_{i j} I_{j}$, then the principal components are $\alpha_{13}, \alpha_{14}, \alpha_{23}, \alpha_{24}$, their invariants of 1 st order being $\alpha_{12}^{2}+\alpha_{14}^{2}+\alpha_{23}^{2}+\alpha_{24}^{2}$, and $\alpha_{13} \alpha_{24}-\alpha_{23} \alpha_{14}$. For the basic figure and the rolling figure these two invariants must be the same. 


\section{Euclidean Motion}

Let the vertex of the frame be $A$, the axes $I_{1}, \ldots, I_{n}$, and let their relative instantaneous motion be $d A=\sum \omega_{i} I_{i}, d I=\Omega I$. If, by the frame transformation $\bar{A}=A+\sum x_{i} I_{i}$ and $\bar{I}=P I, \omega_{i}$ and $\Omega=\left(\omega_{i j}\right)$ are transformed respectively to $\bar{\omega}_{i}$ and $\bar{\Omega}=\left(\bar{\omega}_{i j}\right)$, we have

$$
\omega+x \Omega=\bar{\omega} P, \bar{\Omega} P=P \Omega,
$$

where $\omega=\left(\omega_{1}, \ldots, \omega_{n}\right), \bar{\omega}=\left(\bar{\omega}_{1}, \ldots, \bar{\omega}_{n}\right)$ and $x=\left(x_{1}, \ldots, x_{n}\right)$. Now we take an orthogonal matrix $P$ such that $\bar{\Omega}$ is of the form (2). When $|\Omega| \neq 0$, we can take $x_{1}, \ldots, x_{n}$ such that $\bar{\omega}_{1}=0, \ldots, \bar{\omega}_{n}=0$. When $|\Omega|=0$, we take $\bar{\omega}$ such that (3) can be solved for the unknown $x_{i}$. We perform the above consideration for the case $n=3$. In this case (2) is of the form $\bar{\Omega}=0+\left(\begin{array}{ll}0 & \lambda \\ -\lambda & 0\end{array}\right)$. When $\Omega=0$, our motion is a translation at every instant. Hereafter we treat the case $\Omega \neq 0$, namely $\lambda \neq 0$. Owing to the speciality of dimensions 3 , we have by the second equation of $(3)\left(\Omega=\left(\omega_{i j}\right), P=\left(p_{i j}\right)\right)$,

$$
\begin{gathered}
\omega_{23}=\lambda p_{11}, \quad \omega_{31}=\lambda p_{12}, \quad \omega_{12}=\lambda p_{13}, \\
\lambda^{2}=\omega_{23}^{2}+\omega_{31}^{2}+\omega_{12}^{2} .
\end{gathered}
$$

The first equation of $(3)$ is in this case

$$
\omega_{i}+\sum_{j=1}^{3} x_{j} \omega_{j i}=\sum_{j=1}^{3} p_{j i} \bar{\omega}_{j} \quad(i=1,2,3),
$$

which by (4) gives rise to

$$
\omega_{1} \omega_{23}+\omega_{2} \omega_{31}+\omega_{3} \omega_{12}^{2}=\lambda \bar{\omega}_{1} 。
$$

This is the only condition that (6) should be consistent. So if we take $x_{1}, x_{2}$, $x_{3}$ suitably we have $\bar{\omega}_{2}=0, \bar{\omega}_{3}=0$ except $(7)$.

The line through $\left(x_{1}, x_{2}, x_{3}\right)$ with the direction $\left(p_{11}, p_{12}, p_{13}\right)$ is the instantaneous center, while the basic figure $C_{1}$ and the rolling figure $C_{0}$ are ruled surfaces. If we take along the basic ruled surface the cartesian frame with $I_{1}$ on the generic line, we have

$$
d A=\sum \alpha_{i} I_{i}, \quad d I_{i}=\sum \alpha_{i j} I_{j},
$$

where $\alpha_{2}, \alpha_{3}, \alpha_{12}, \alpha_{13}$ are principal components. When we transform this to the Frenet's frame, we have ([1], p. 51)

(9) $d A=d \sigma\left(\alpha I_{1}+k I_{3}\right), \quad d I_{1}=d \sigma \cdot I_{2}, \quad d I_{2}=d \sigma\left(-I_{1}+\beta I_{3}\right), d I_{3}=d \sigma\left(-\beta I_{2}\right)$.

The relative components of $T_{a^{-1}} \cdot \delta S \cdot T_{a}$ is $\bar{\omega}_{1}$ and $\lambda$, and we have

$$
d A=\bar{\omega}_{1} I_{1}, d I_{1}=0, d I_{2}=\lambda I_{3}, d I_{3}=-\lambda I_{2} .
$$

So for the basic ruled surface and the rolling one, do and $k d \sigma$ are the same, while $\alpha$ and $\beta$ are in general different.

If $\omega_{1} \omega_{23}+\omega_{2} \omega_{31}+\omega_{3} \omega_{13}=0$, then by $(7) \bar{\omega}_{1}=0$, and by (10) there is no 
slipping on the generic lines of the two surfaces, $\alpha$ being the same, which shows that the lines of striction of the two surfaces coinside. When the two surfaces are developable, namely $k=0$, we have, from (9), $d A=\alpha d \sigma \cdot I_{1}, d I_{1}=d \sigma \cdot I_{2}$ and $\alpha$ is the radius of curvature of the edge of regression of the surface. So in the case of developable surfaces the condition $\bar{\omega}_{1}=0$ reduces to the coincidence of the radius of curvature of the edge of regression. A spherical motion can be characterised by the relation $k=0$ and $\alpha=0$.

Now we represent the invariants of our basic ruled surface by the relative component of our motion. We write $\left(p_{1}, p_{2}, p_{3}\right)$ in place of $\left(p_{11}, p_{12}, p_{13}\right)$, which are by (4)

$$
p_{1}=\omega_{23} / \lambda, \quad p_{2}=\omega_{31} / \lambda, \quad p_{3}=\omega_{12} / \lambda \quad\left(\lambda^{2}=\omega_{23}^{2}+\omega_{31}^{2}+\omega_{12}^{2}\right) .
$$

By (7) we have

$$
\bar{\omega}_{1}=(1 / \lambda)\left(\omega_{1} \omega_{23}+\omega_{2} \omega_{31}+\omega_{3} \omega_{12}\right), \quad \bar{\omega}_{2}=0, \quad \bar{\omega}_{3}=0 .
$$

Hence (6) can be written as

$$
r_{1}-p_{3} x_{2}+p_{2} x_{3}=0, \quad r_{2}+p_{3} x_{1}-p_{1} x_{3}=0, \quad r_{3}-p_{2} x_{1}+p_{1} x_{2}=0,
$$

where we put

$$
r_{i}=(1 / \lambda)\left(\omega_{i}-p_{i} \bar{\omega}_{1}\right) \quad(i=1,2,3) .
$$

Then by the consideration of (8), (9), (11), (13) and the process [1] p. 49, 50 we get the following: (calculation being omitted,)

$$
\begin{aligned}
& d \sigma^{2}=\alpha_{12}^{2}+\alpha_{13}^{2}=d p_{1}^{2}+d p_{2}^{2}+d p_{3}^{2}, \\
& k d \sigma^{2}=\alpha_{2} \alpha_{13}-\alpha_{3} \alpha_{12}=\left|\begin{array}{lll}
p_{1} & d p_{1} & d x_{1} \\
p_{2} & d p_{2} & d x_{2} \\
p_{3} & d p_{3} & d x_{3}
\end{array}\right|=d p_{1} d r_{1}+d p_{2} d r_{2}+d p_{3} d r_{3}, \\
& \alpha d \sigma=\alpha_{1}-d\left(\frac{\alpha_{2} \alpha_{12}+\alpha_{3} \alpha_{13}}{\alpha_{12}^{2}+\alpha_{13}^{2}-}\right)=p_{1} d x_{1}+p_{2} d x_{2}+p_{3} d x_{3}-d\left(\frac{d x_{1} d p_{1}+d x_{2} d p_{2}+d x_{3} d p_{3}}{d p_{1}^{2}+d p_{2}^{2}+d p_{3}^{2}}\right) \\
& =-\left|\begin{array}{lll}
d p_{1} & p_{1} & r_{1} \\
d p_{2} & p_{2} & r_{2} \\
d p_{3} & p_{3} & r_{3}
\end{array}\right|-d\left(\left|\begin{array}{lll}
d p_{1} & p_{1} & d r_{1} \\
d p_{2} & p_{2} & d r_{2} \\
d p_{3} & p_{3} & d r_{3}
\end{array}\right| /\left(d p_{1}^{2}+d p_{2}^{2}+d p_{3}^{2}\right)\right), \\
& 3 d_{\sigma}=\alpha_{23}+d \tan ^{-1} \frac{\alpha_{13}}{\alpha_{12}}=\left|\begin{array}{lll}
p_{1} & d p_{1} & d^{2} p_{1} \\
p_{2} & d p_{2} & d^{2} p_{2} \\
p_{3} & d p_{3} & d^{2} p_{3}
\end{array}\right| /\left(d p_{1}^{\mathrm{a}}+d p_{2}^{2}+d p_{3}^{2}\right) .
\end{aligned}
$$

\section{Projective Motion (complex number field)}

Let the frame be $A_{0}, A_{1}, \ldots, A_{n}$ and the instantaneous relative displacement be $d A_{i}=\sum_{j=0}^{n} \omega_{i j} A_{j}(i=0, \ldots, n)$, which we write briefly $d A=\Omega A$. By 
the frame transformation $\bar{A}=T A \Omega$ is transformed to $\bar{Q}$, which is given by $\bar{Q}$ $=T \Omega T^{-1}$. Here we assume that $\Omega$ has only simple elementary divisors. Then for suitably chosen $T$, we have $\bar{\Omega}=\alpha_{0} \dot{+} \alpha_{1} \dot{+} \ldots \dot{+} \alpha_{n}$, and our instantaneous center is the set of $n+1$ points $A_{0}, \ldots, A_{n}$. For the one-parametric figure of these sets of $n+1$ points, we attach the frame $A_{0} A_{1} \ldots A_{n}$, for which $d A_{i}$ $=\sum \alpha_{i j} A_{j}$. When $\alpha_{i 0} \neq 0, \alpha_{0 i} \neq 0(i \neq 0)$, we see that the complete system of invariants is the following,

$$
\alpha_{0 i} \alpha_{i 0}(i \neq 0), \quad \alpha_{0 i} \alpha_{j 0} \alpha_{i j}(i \neq j \neq 0) .
$$

We omit here the details. Our motion is the one, by which two figures of oneparametric set of $n+1$ points, for which (15) are the same, are basic and rolling figures.

\section{Nagoya University}

